\title{
Automation of Billing System for Smart Parking of Vehicles at Institutions
}

\author{
Dhruva Hunusekatte \\ PES University, South Campus, India
}

\begin{abstract}
A system is proposed with least human intervention to achieve an efficient billing system for a parking lot. By managing an efficient and automated billing system, the waiting time of each user is reduced, and thereby allowing the user to have a seamless and hassle-free experience. It also helps in lowering the amount of daily vehicle emissions by reducing the waiting period. This would help in efficient use of fuel, causing less pollution around the Institution's Parking lot, reducing human intervention to the minimum level possible. This system is implemented on an ARM CORTEX M3 Board which has a low power consumption and functions in real time connected with an ESP-8266, MySQL (an opensource database management system), RFID tags, a reader module and a GSM module for mobile communication.
\end{abstract}

\section{Introduction}

At present, the manual parking system used at Institutions consumes time and is not efficient for easy movement of vehicles. Today, people prefer automatic technology to meet their needs. The amount of traffic in recent years has been steadily increasing due to the increasing number of vehicles.

The focus of this paper is on automating the billing mechanism of the parking area at present, which is done manually, is erroneous, time consuming and hectic to be done on a daily basis. This paper intends to cut down the time consumed by users and provide an efficient billing system which will reduce the human intervention to the minimum level possible. We wish to make the billing system realtime and cashless, which will further save time. It makes the overall system user-friendly.

\section{Related Work and Purpose of Paper}

All printed Referring to some of the previous papers in which automation of parking is done such as using image processing [1], using technologies like Bluetooth and Arduino [2], using RFID based system [3]. But some of the drawbacks faced are number plate recognition is costly and expensive, prone to errors, absence of an efficient and user-friendly billing system with communication of transaction details [4], maximum range of devices such as Bluetooth. The drawbacks were acknowledge and remove using, RFID based system which is more efficient and less error prone, a GSM module for communication of transaction details and a database management system with web application for an efficient and transparent billing system.

The basic idea behind implementing RFID Based Toll System is to automate the toll collection process at institutions parking lots by reducing manual operation in toll booths and also the long queues at toll booths using RFID tags installed on the vehicles. In addition, it can not only help the vehicle owners and system administrators from vehicle theft detection but also help in efficient, hassle free monetary transaction for the user. The following points indicate the advantages of using a completely automated parking system:

- Avoid fuel loss

- Saving of time in collecting toll

- Avoid financial loss

- To monitor the traffic at the toll plaza

- Problems with pursuing toll evaders

- Theft detection

The smart parking system proposed is implemented using an arm microcontroller LPC1768 which is interfaced with an RFID module, a GSM module and also a Wi-Fi module which is connected to a server for organized management of user information.

\section{Design}

Overview of the block diagram in Figure 1 are explain below

\subsection{RFID Reader Module (EM-18)}

It is used to uniquely identify and categorize different objects through the usage of radio frequency waves. It uses an RFID tag and shares data with it seamlessly and instantaneously. It has pins for Vcc, 
gnd, LED/buzz, data pins like data0, data1,Transmitter(tx) and select pin(sel). This device works on UART protocol- transmitter pin can be directly con- nected to microcontroller at receiver pin.

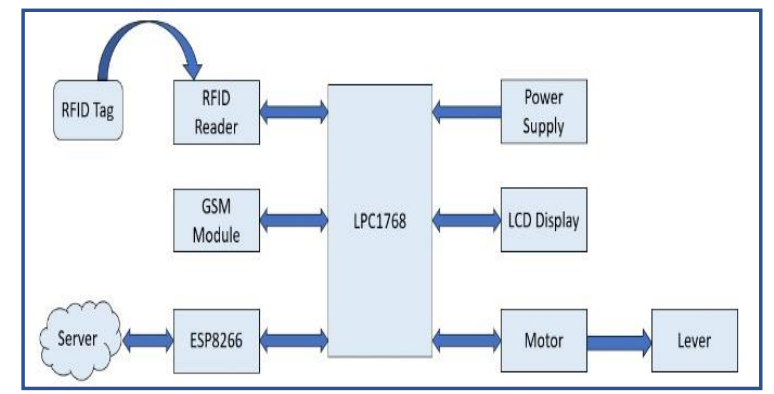

Figure 1. Block Diagram

RFID reader module has a RF signal generator for sending signals to send signals to the tag and also a receiver/ signal detector for receiving signals from the tag. A Reader consists of a Radio Frequency module and an antenna which generates high frequency electromagnetic field waves. [5].

Global System for Mobile (GSM) communication is used to transfer text messages from a phone to another, using the former's sim card. It was created to describe the protocols for second-generation (2G) digital cellular networks. The advantages of using GSM are but not limited to the following:

- Extensive Coverage: Extensive use throughout the world

- Greater Phone Variety: Most phones operate on GSM

- Supports GPRS (General Packet Radio Services)

- Supports SMS (Short message service)

\subsection{Arm Cortex M3 microcontroller LPC1768}

The ARM Cortex-M is a group of 32-bit RISC ARM controller cores licensed by Arm Holdings. These cores are optimized for low-cost and energyefficient microcontrollers, which have been embedded in tens of billions of consumer devices [6]. It uses thumb 2 architecture set, which is 25 percent faster than thumb architecture set is very efficiency. It uses Cortex-M3 which is faster than its predecessors. It has pipelining which enhances the system speed.

The Lower Power Consumption (LPC1768) can efficiently manage the power consumption through its 4 low power modes namely sleep mode, deep sleep mode, power down mode, deep power down mode.

\subsection{ESP8266}

The ESP8266 a System on a Chip (SoC), manufactured by the Chinese company Espressif Systems. It consists of a Tensilica L106 32-bit micro controller unit (MCU) and a Wi-Fi transceiver. It has 11 GPIO pins (General Purpose Input/Output pins), and an analog input as well [7].

\subsection{Software Used}

MySQL is an Oracle-backed open source relational database management system (RDBMS) based on Structured Query Language (SQL). MySQL runs on virtually all platforms, including Linux, UNIX and Windows. Although it can be used in a wide range of applications, MySQL is most often associated with web applications and online publishing [8].

The web browser is considered a software application that allows people to access, retrieve and view information on the internet. The web browser retrieves the necessary content from a web server and then displays the resulting web page on the user's device. The information that may be "browsed" can be in the form of text content on a web page, an image, video, audio [9],[10].

Keil is a popular software development environment for ARM Cortex-M based Microcontroller devices based on the instruction set available. The Vision Device Database automatically configures the development tools for the target microcontroller. Vision incorporates project manager, editor, and debugger in a single environment.

Django is a Python-based free and open-source web framework that follows the model-template-view (MTV) architectural pattern. It is maintained by the Django Software Foundation (DSF), an American independent organization established as a non-profit. Django's primary goal is to ease the creation of complex, database-driven websites. The framework emphasizes reusability and "pluggability" of components, less code, low coupling, rapid development, and the principle of do not repeat yourself.

\section{Implementation}

The GSM module and RFID reader module transmit and receive data to the processor serially using UART as communication protocol, and the LCD is interfaced with the microcontroller using GPIO pins. The Block diagram depicted in Figure 1 is implemented in the following way:

i. The GSM module, EM-18 reader module and the LCD display are interfaced with LPC1768 (set to low power mode) work as an efficient system in this project. 
ii. The RFID tag is scanned by the EM18 reader module, data bits are produced which are sent to the LPC18 as a bit stream. On receiving the bit stream, the LPC1768 sends it to the server through the node MCU ESP -8266 using an URL. The data bit stream is used to identify the unique ID of the RFID tag.

iii. The server identifies the unique ID and updates the transaction details in its database. Once the transaction is completed, server sends a response back to the microcontroller.

iv. The microcontroller instructs the GSM module to send the billing details as a SMS to the user, and at the same time displays basic user data such as vehicle type, amount and time of transaction on the LCD. The user can login to the website and see all the information related to the transaction, communication protocol, and the LCD is interfaced with the microcontroller using GPIO pins.

The Figure 2 represents the basic flow of the implementation of the project. The sequence of steps assumed that all users are registered users:

\section{Step 1: Start}

\section{Step 2: Detect the RFID tag}

Step 3: Deduct the balance and update the details on the database

Step 4: On successful completion of the payment send an SMS of billing details using GSM module to the user and display user

\section{Step 5: End}

\subsection{Assumptions and Requirements}

All the users that are entering the parking lot are required to be registered users. After the Implementation is carried out, the following results were obtained. The Figure 3 shows the interfacing of LPC1768 with EM-18 reader module, GSM module and ESP8266. ESP8266 is connected to the college parking lot server named "Automatic Parking Toll Collection". The users will be given an RFID card of their own which they will be expected to carry whenever they want to park their vehicle in the parking lot. Upon placing the card in front of the EM 18 Card reader. Once the balance is updated, the server contacts the Wi-Fi module which is interfaced with the LPC. The LPC on receiving the information from ESP8266, sends a command to the GSM module to send a message to the respective user of the updated balance. The user has access to the server through internet and a web browser and can open his account at any point of time to check his updated balance.

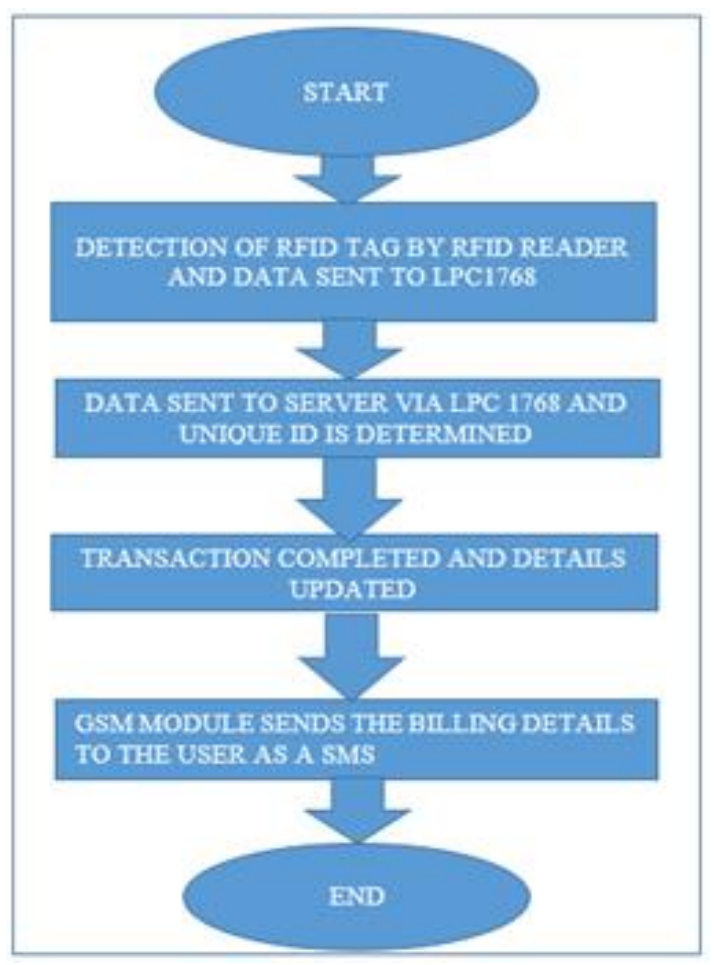

Figure 2. Flowchart of the process

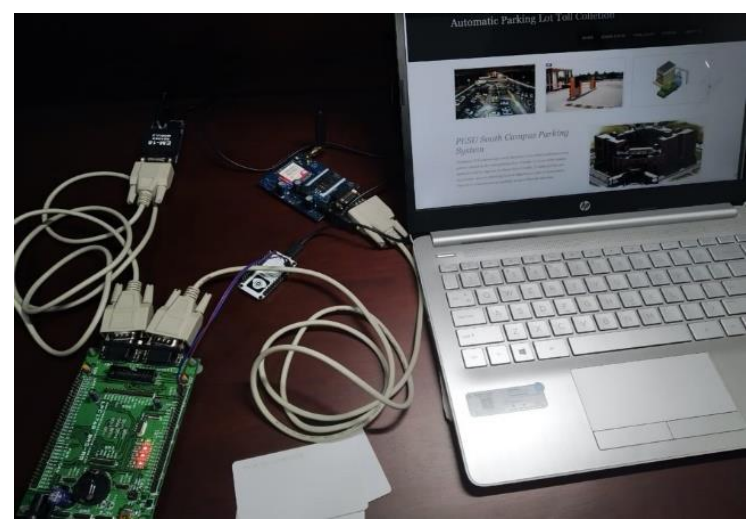

Figure 3. Picture depicting all the interfaces

The Figure 5 shows the server data after the user has placed the RFID card in front of the reader and the user is identified as an authenticated user with updated balance. In this case, the user is user no. 10 whose account balance has been deducted by Rs .10. The transaction SMS is sent to the user's registered phone number by the GSM module, after the transaction is completed. The GSM is used to track theft of vehicle and as a log for the user to see his/her transaction details every time the person uses the parking lot. The Web service is used to enhance the user's (admins and 
parking lot users) experience and give transparency to the whole system.

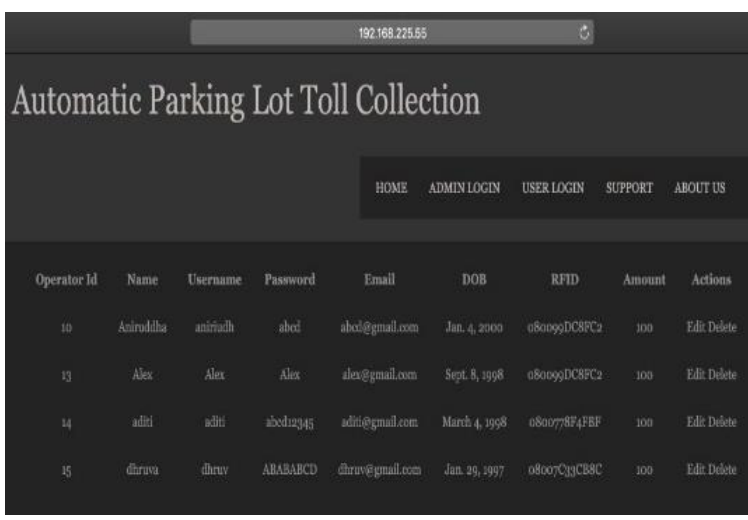

Figure 4. User's server data

(before placing the RFID card in front of the reader)

After the transaction, the user is allowed to park his vehicle inside the parking lot.

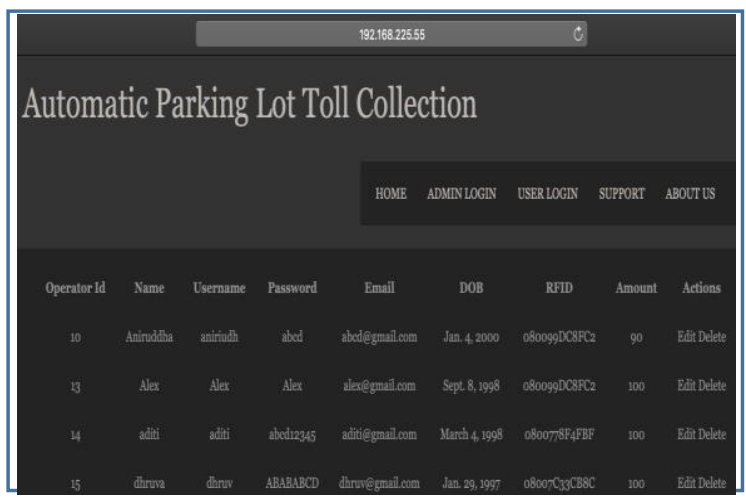

Figure 5. User's data in the server after transaction

In cases where the user is not identified as an authenticated user or there is not enough balance, the user will be instructed to add money to his account to enable a successful transaction.

\section{Results and Observation}

The experiment was conducted on 15 vehicles for 5 days, in which 15 vehicles arrived at the same time and average waiting time for each was obtained. Waiting time is significantly large for vehicles arriving last, which led to noise pollution. As seen in the Figure 6 graph, there is significant waiting time for the vehicle arriving last. When used the automated parking system as compared to manual, the waiting time is reduced from 225 seconds to nearly 120 seconds for the 15 th vehicle.

During peak hours at the Institution, there were large no of vehicles (approximately 20 vehicles are waiting in a line). This caused unnecessary fuel consumption of these vehicles (0.6 liter / hour is consumed by idle vehicles) causing air pollution at the Institution.

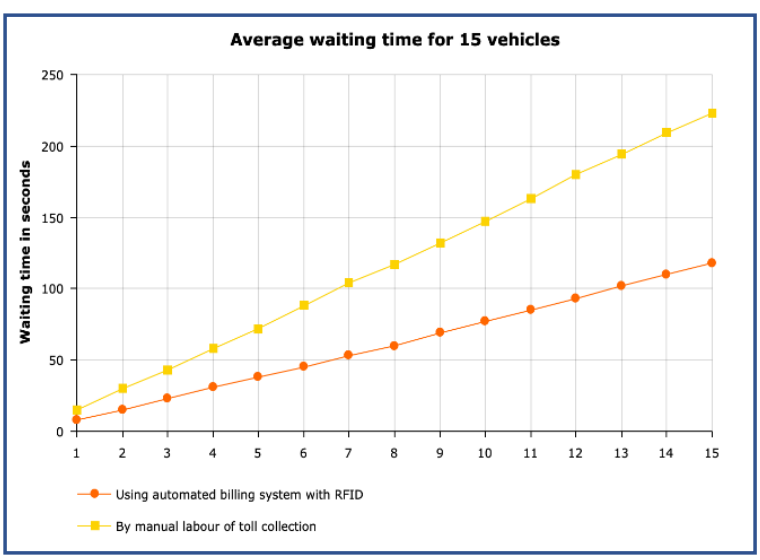

Figure 6. Time taken by using different systems for parking at institution (Manual and Automated).

The latter resulted in the $\mathrm{Co} 2$ emission to drop from $1000 \mathrm{ppm}$ to $700 \mathrm{ppm}$ on average readings across 5 days. Using this system, the pollution levels can be significantly decreased at parking lots across all Institutions

\section{Conclusion}

In this paper, a system is proposed to cut down the waiting period experienced by users at parking lots in Institutions by making an efficient cashless and completely automated billing system with enhanced security and user experience. The reader identified the user as an authenticated user and the amount will be deducted from his/her balance. By assigning a unique RFID card to each user and the amount details the reader will be able to detect each user and update the details on the server and thereby speeding up the billing process, and also help in the complete automation of the entire process.

An efficient billing system is implemented which provides transparency with help of web applications and GSM module providing maximum convenience to the user by providing transaction details and also helps in theft monitoring of vehicles. The above project can be implemented in all parking lots, thereby automating the entire process.

\section{References}

[1] M. M. Rashid, A. Musa, M. Ataur Rahman, and N. Farahana, A. Farhana (2012). Automatic Parking Management System Based on Number Plate Recognition, 'International Journal of Machine Learning and Computing, Vol. 2, No .2, April. 
[2] M. Geetha, B. Sangeetha, J.Vidhya, S. Ramya, L.N.K. Ragavi (2015). Automatic tollgate payment system using Hybrid Mechanism, Journal of Chemical and Pharmaceutical Sciences.

[3] Satyasrikanth P, Mahaveer Penna, Dileep Reddy Bolla, (2016). Automatic toll collection system using RFID, International Journal of Computer Science and Mobile Computing, ISSN: 2320-088X.

[4] Oindrila Chakraborty, Nalini N., (2017). Automated Toll and Parking Systems, International Journal of Advanced Research in Computer Science and Software Engineering, ISSN: 2277-128X.

[5] Electronicwings.com 'Rfid card reader, https:// www.electronicwings.com/sensors-modules/rffid-readerem 18 (Access Date: 15 Feb 2020).

[6] Nxp.com 'Microcontroller ARM Cortex m3, https://www.nxp.com/products/processors-andmicrocontro llers/arm-microcontrollers/generalpurpose-mcus/lpc1700cortex-m3/512kb-flash-64kb-sram-ethernet-usb-tfbga100p ackage:LPC1768FET100 (Access Date: 5 February, 2020).

[7]https://www.espressif.com/en/products/hardware/esp82 66ex/overview' 'ESP 8266 reference', (Access Date: 15 February, 2020).

[8] MySQL.com, An open source database management system, https://www.mysql.com/ used in this paper, (Access Date: 15 February, 2020).

[9] Udemy, For basics of HTML and CFF, https:// www.udemy.com/ (Access Date: 15 February, 2020).

[10] Django Project, For having a rough overview on basics of Django, https://docs.djangoproject.com/en/2.2/ (Access Date: 15 February, 2020).

\section{Acknowledgements}

I cannot express enough thanks to IEEE ICITST committee for their continued support. I offer my sincere appreciation for the learning and conference opportunities provided. My completion of the project could not have been accomplished without the support of my classmates, Alex Jose, Aditi C, Aniruddha Banerjee - Thank you for your support. 